\title{
Specimen Preparation of Difficult Materials with Patterned Dimpling Wheels
}

\author{
M. P. Moran, A. K. Norman and D. M. Follstaedt \\ Physical and Chemical Sciences, Sandia National Laboratories, Albuquerque, NM 87185-1056
}

A faster, gentler means is needed for mechanical thinning of fragile specimens for transmission electron microscopy (TEM) to avoid fracture. As described in an accompanying paper (Follstaedt et al.), we have developed patterned dimpling wheels that achieve both these objectives. Here we describe using these wheels to thin two very different, but difficult, materials: sapphire and InP.

Sapphire is very slow to grind without resorting to harsh methods that promote fracture at thicknesses less than $20 \mu \mathrm{m}$. Very slow to erode in the ion mill, such thick specimens require long milling times that can lead to exaggerated surface topography. For cross-section specimens with other supporting materials (Si spacers, glue) holes can occur in other places, allowing redeposition.

We have prepared a plan-view specimen of a $3.2 \mu \mathrm{m}$ GaN layer grown on a $450 \mu \mathrm{m}$ sapphire wafer. A $3 \mathrm{~mm}$ diameter disc was ultrasonically cored from the wafer and mounted layer down on a pedestal for dimpling with a Fischione Model 2000 unit. First, $320 \mu \mathrm{m}$ of the sapphire substrate was removed in $50 \mathrm{~min}$. using a slotted stainless steel flattening wheel and $15 \mu \mathrm{m}$ diamond paste. An additional $30 \mu \mathrm{m}$ was removed in $20 \mathrm{~min}$. with the same wheel carrying $6 \mu \mathrm{m}$ diamond compound (103 $\mu \mathrm{m}$ remaining thickness). The specimen was then dimpled to a depth of $56 \mu \mathrm{m}$ in $70 \mathrm{~min}$. using a slotted stainless steel wheel and $1 \mu \mathrm{m}$ diamond compound. An additional $37 \mu \mathrm{m}$ of sapphire was removed in $160 \mathrm{~min}$. using a patterned Delrin wheel, initially carrying $3 \mu \mathrm{m}$ diamond paste but with the last $4 \mu \mathrm{m}$ using $1 \mu \mathrm{m}$ diamond. The entire flattening / dimpling procedure was performed using a $40 \mathrm{~g}$ load, a wheel speed of 2 RPM and a table rotation speed of 1 RPM. The final thickness was determined optically to be $\sim 10 \mu \mathrm{m}$ with a high-resolution microscope at $1000 \mathrm{x}$ by focusing on features on both sides of the transparent specimen. The specimen was ion milled for $25 \mathrm{~min}$. to perforation in a Gatan Model 691 PIPS with two guns using argon at $3.5 \mathrm{keV}$ and $5^{\circ}$ incident beam angle. The resulting electron transparent material; a band approximately $35 \mu \mathrm{m}$ wide around the hole, was usable for examining vertical threading dislocations in the GaN, as seen in Fig. 1.

A double-sided cross-sectional specimen of the same material was prepared using Si backing slabs and well known methods to produce a planar disk $115 \mu \mathrm{m}$ thick. The disk was dimpled from both sides using a Gatan Model 656 unit with a patterned Delrin wheel and $1 \mu \mathrm{m}$ diamond. The final thickness of $5 \mu \mathrm{m}$ was achieved in $4 \mathrm{hrs}$. Perforation occurred at the glueline after milling $50 \mathrm{~min}$. at $3^{\circ}$ beam incidence. Threading dislocations could be studied on both sides of the glueline over distances of $30 \mu \mathrm{m}$ or more from the hole, as in Fig. 2. This result shows that patterned Delrin wheels are effective even on very hard materials, whereas unpatterned wheels are very ineffective.

InP is much softer than sapphire but also prone to fracture. An $80 \mu \mathrm{m}$-thick cross-section disk of modulated InAlAs grown on InP was dimpled to 3-5 $\mu \mathrm{m}$ in 1 hour with a patterned Delrin wheel and $1 \mu \mathrm{m}$ diamond. Perforation occurred after milling 10 minutes using $3.5 \mathrm{keV}$ Ar at $4^{\circ}$. Extended thin area along the glueline (Fig. 3) allowed $30 \mathrm{~nm}$ composition modulations to be studied (inset).

Patterning the wheel surfaces not only increases removal rate but offers additional control by making gentler treatments and softer wheel materials (Delrin) more effective. Patterned wheels also retain polishing compound better and require less attention during dimpling. The thinner specimens obtained with patterned Delrin wheels provide greater thin area for TEM examination. 


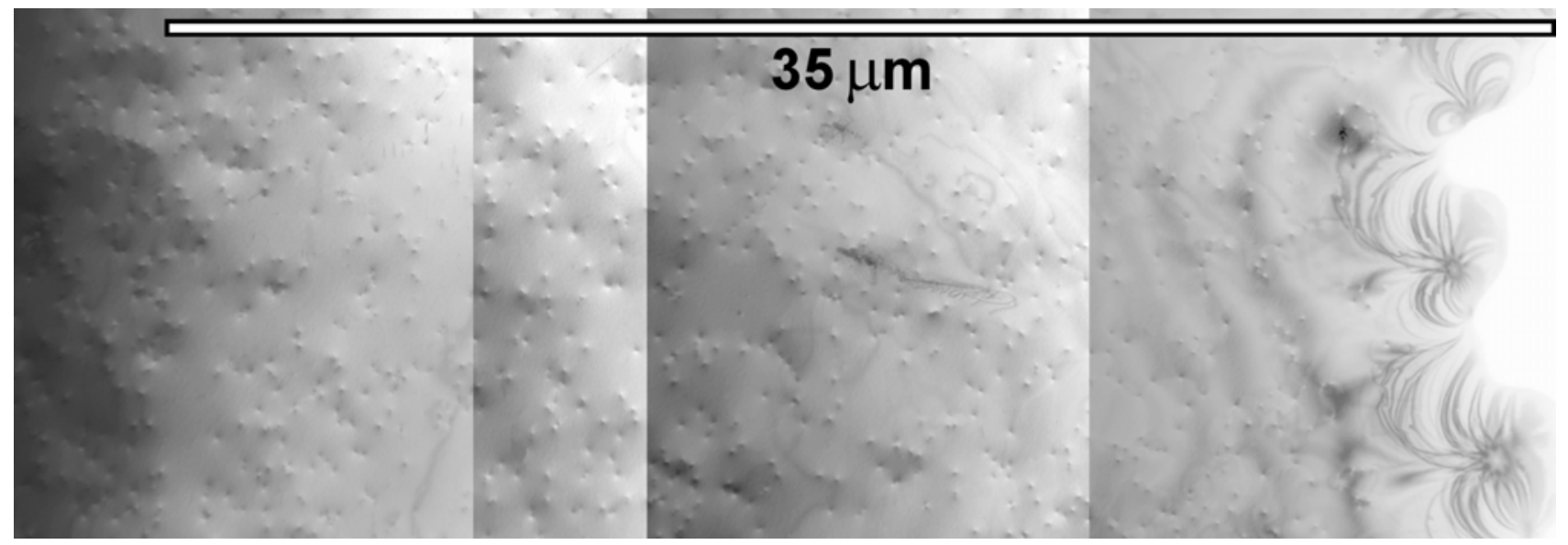

Figure 1. Bright-field, plan-view image of vertical threading dislocations (small spots) at the (0001) surface of $\mathrm{GaN}$ grown on sapphire. Obtained with (11-20) two-beam diffraction conditions.

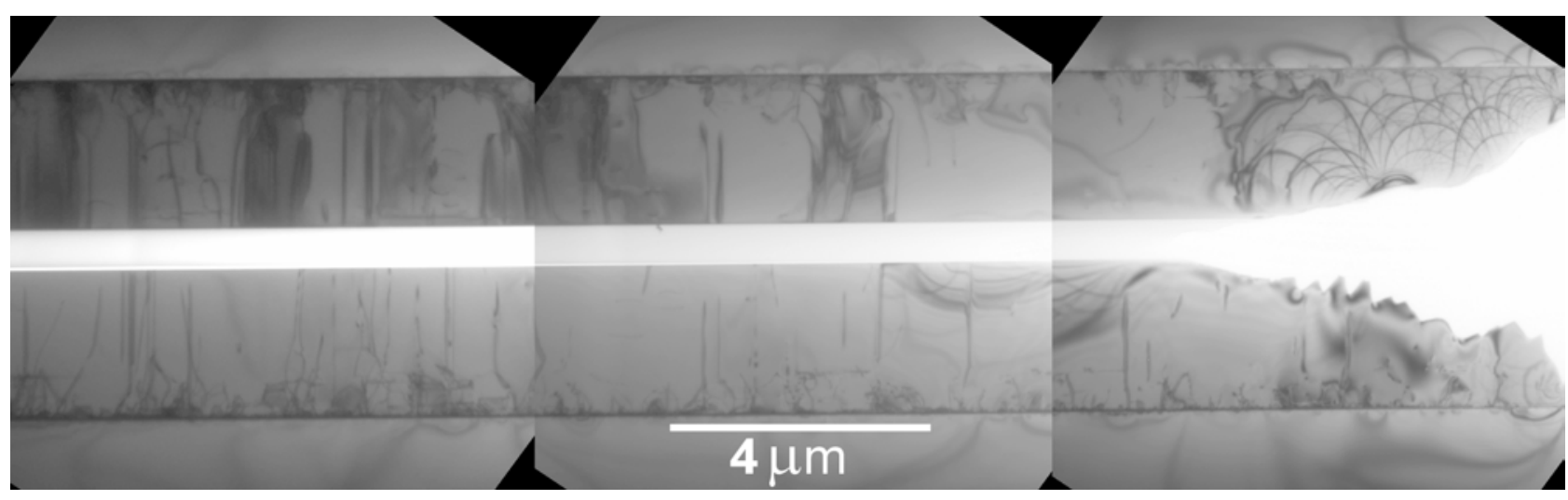

Figure 2. Cross-sectional view of threading dislocations in GaN grown on sapphire. The specimen shown is a dual cross-section with the GaN epi-layers epoxied face to-face.

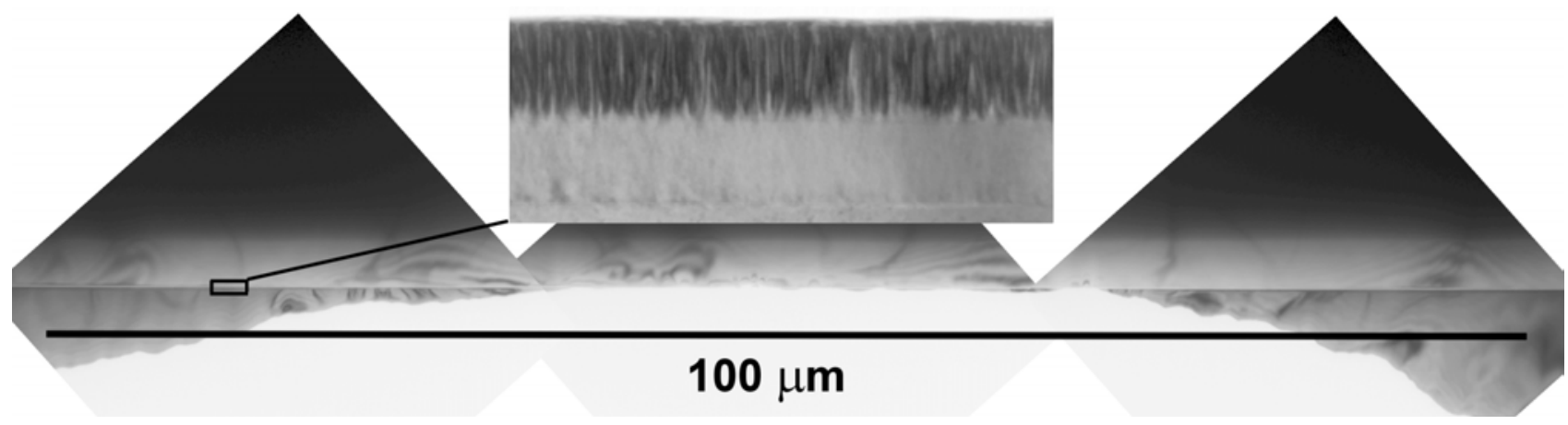

Figure 3. Cross-section view of composition modulations in a InAs / AlAs superlattice. A $100 \mathrm{~nm}$ InAlAs alloy buffer was first grown on the InP substrate, followed by the $100 \mathrm{~nm}$ superlattice. The enlargement shows the modulated structure and the alloy buffer in the area indicated with the box.

Sandia is a multi-program laboratory operated by Sandia Corporation, a Lockheed Martin Company, for the United States Department of Energy under contract DE-AC04-94-AL85000. 